\title{
Stress Tensor in Granular Media and in other Mechanical Collections
}

\author{
J. J. MOREAU \\ Laboratoire de Mécanique et Génie Civil \\ Case 048, Université Montpellier II, 34095 Montpellier Cedex 5, France.
}

May 5, 2005

\begin{abstract}
Instead of the pair-by-pair approach conventionally used when defining the average stress tensor of a granular sample or of a piece of masonry, a grain-by-grain construction is proposed. Its theoretical foundation lies in the assignement, to any bounded mechanical system, of the tensor moment of its internal efforts, shortly called the internal moment of the system. In classical continuous media, the Cauchy stress is nothing but the volume-density of internal moment (or its negative, depending on the sign conventions made). This approach is not limited to systems in equilibrium; furthermore, it applies to collections involving other mechanical objects than massive grains. The pertinence of the concept is demonstrated first by numerical simulations. Secondly some mathematical procedures of smoothing and homogenisation are introduced to connect the microscopic analysis with the macroscopic continuum model. Quantitatively, in usual situations, what is obtained as stress tensor differs very little from the value resulting from traditional definitions.
\end{abstract}

\section{Introduction}

\subsection{Motivation}

In many situations of Civil Engineering or Geomechanics soil materials, though perceptibly granular, are treated in the framework of classical Continuum Mechanics, with the Cauchy stress field as central concept. The crucial problem in this macroscopic treatment, whose consistency remains out of question, is that of determining the constitutive laws which should connect this macroscopic stress field with some descriptors of the material evolution.

One popular tool in the investigation of granular behaviour at the microscopic level is numerical simulation in which the laws of mechanics are applied to individual grains and to their interactions. Simulations may be convincingly validated through comparison with some experiments which provide a view of grain motions [9][23] or by checking the quantitative agreement between the measurements of some experimentally accessible quantitities and the corresponding numerical evaluations. Under such a validation, one of the benefits of computation is the assessment of microscopic quantities, e.g. the grain-to-grain contact forces, very uneasy to measure in real world experiments. Connecting these quantities with the elements involved in the macroscopic treatment is a non trivial task. This paper is meant to provide some tools on this purpose.

Let $\mathrm{D}$ denote a probe domain in the space of a simulation, containing an exact number of grains. Some authors (e.g.[11][3]) have observed that, from the strict standpoint of vocabulary, invoking the average stress tensor of $\mathrm{D}$ does not rest on any specific definition: this merely is the volume average over $\mathrm{D}$ of the tensor function $\boldsymbol{\sigma}$, the Cauchy stress, defined in the material each 
grain is made of and equal to zero in the interstitial space (or evaluated in some fluid possibly filling this space; see Parag. 3.2 below). This average, i.e. $\int_{\mathrm{D}} \boldsymbol{\sigma} d v / \operatorname{vol}(\mathrm{D})$, has naturally to be calculated by totalizing the respective contributions of each individual grain to the integral. At this stage one receives as a piece of luck that the contribution of a grain, say B, may be evaluated without any continuum mechanics analysis. In fact, in the case of equilibrium, some standard formula (equ.(14) in the sequel) connects $\int_{\mathrm{B}} \boldsymbol{\sigma} d v$ with the tensor moment of the external forces that $\mathrm{B}$ experiences. These forces may come from neighbouring grains or from boundaries or also be distant forces as the gravitational ones. In numerical simulation, computing the average stress over D is thus straightforward. It is shown in Sec. 4 how this type of approach extends to non-equilibrium situations.

In spite of the above elementary properties, the majority of the authors dealing with the subject follow a different route. Accompanied with diverse comments, the common trend is to select in each grain a reference point, say $\boldsymbol{r}$ and $\boldsymbol{r}^{\prime}$ for two grains B and B'. If $\boldsymbol{f}$ is a contact force exerted by B' on B, the tensor $\left(\boldsymbol{r}^{\prime}-\boldsymbol{r}\right) \otimes \boldsymbol{f}$ is counted in a total involving all contact points present in the probe domain. In Parag. 3.4 below, where the uncertainty arising from the arbitrariness in the choice of reference points is assessed, this is called the pair-by-pair approach. It seems that these authors have been induced to adopt such a standpoint by historical references. In fact, in the original work of Cauchy, the concept of stress arised from the study of a model of crystalline matter consisting of a lattice of material points exerting forces upon each other, clearly leading to pair-by-pair calculations.

It was observed [4] that the differences produced by changing reference points are of the same order of magnitude as the terms alleged to reveal a possible lack of symmetry of the tensor, a controversial question. See also a discussion of such uncertainties in [6].

In contrast, the theoretical consistency of the grain-by-grain approach proposed in this paper, allowing for its extension to more general assemblies than common granulates and to some dynamic regimes, is emphasized in this paper. As an advantage, let us mention the possibility of an analysing the spatial diversity of grain stress conditions (see e.g.[44]). On Fig.** in the sequel, the stress integral of each grain in a two-dimensional simulation is represented by a cross figuring its principal directions and eigenvalues. This is a way among others of making visible the popular force chains.

Putting it bluntly, the author views the use of reference points as a counterproductive detour. But, in all the static or quasi-static circumstances tested so far, the difference between the alleged stress tensors respectively obtained by the conventional methods and by that presented here are found very small. So the present paper does not tend to invalidate the numerical results published elsewhere.

\subsection{The theoretical background}

One may object to the construction sketched above the reference made to the Cauchy stress field in each grain, while this field actually disappears from the final expressions. Anyway, such a reference cannot apply to collections involving some objects too singular for possessing a Cauchy stress field. In particular, it has no relevance to the assemblies of ideal points subject to interaction potentials, modelling crystalline matter, as considered from the time of Cauchy to that of Love [29].

In the sequel, a deeper insight and a wider scope are gained by following the route opened in [36]. To prevent misconceptions, one refrains from using the word "stress"; the central concept will be the internal moment, i.e. the tensor moment of the internal efforts of a (bounded) material system, whose existence is secured when the principles of Classical Mechanics are formulated in terms of virtual power (see Section 2 below).

In the case of a portion of standard continuous medium, such as B above, the internal 
moment is found equal to $-\int_{\mathrm{B}} \boldsymbol{\sigma} d v$. In other words, the tensor field $-\boldsymbol{\sigma}$ constitutes the density of internal moment relative to the volume (i.e. Lebesgue) measure $d v$.

This is the place for observing that, when a partial differential equation involving the stress field of a continuum is understood "in the sense of Distributions", the mathematical object which properly constitutes a Schwartz Distribution is not the function $\boldsymbol{x} \mapsto \boldsymbol{\sigma}(\boldsymbol{x})$ but the tensor-valued measure $\boldsymbol{\sigma} d v$, more precisely its negative, that we propose to call the internal moment measure. The minus sign there introduced reflects the common habit in Mechanical Engineering and general Continuum Mechanics of counting a stress as positive when it is directed as a traction. It should be removed if the reverse convention, usual in Civil Engineering and Geomechanics, were made.

It is a principle of Classical Mechanics that the internal efforts of a mechanical system have zero total virtual power whenever the chosen virtual velocity field is that of a rigid motion. Equivalently, the internal moment tensor of any system is independent of the choice of an origin and symmetric. This still holds true if the system experiences as external efforts some distributions of magnetic torques or the point torques introduced in rigid body dynamics for modelling the resistance to rolling (see Parag. 2.4 below).

In addition to proper grains, the internal moment tensor is also defined for such objects as fragments of shells, membranes, threads, etc.. This makes the present construction applicable, for instance, to the mix of sand and flexible threads called Texsol [24][26].

The internal moment of a collection of mechanical objects which interact only through contact equals the sum of their respective internal moments. In fact, for a pair of contacting objects B and B', the contact efforts exerted by B upon B' and those exerted by B' upon B are localized at the same points of space and opposite, by virtue of the action-reaction principle. The total of their moment tensors is thus zero.

In contrast, if the grains of a collection exert distant actions upon each other, the additivity of internal moments doesn't hold true anymore. For instance, authors studying unsaturated soils commonly model the capillary bridges that interstitial water may build between two grains B and B' by a pair of forces that a point $\boldsymbol{b}$ of B and a point $\boldsymbol{b}^{\prime}$ of B' exert upon each other. Such an interaction bridge possesses an internal moment, to be added to the internal moments of other members of the collection (see Parag.2.6 below) to obtain the internal moment of the whole. In Cauchy's case of a system of ideal points subject to distant interactions, the internal moment of the system entirely comes from such bridges.

\subsection{Dynamics}

If the considered mechanical system is in equilibrium, relative to some Galilean frame, the Principle of Virtuel Power implies that the sum of its external and internal moment tensors is zero. In particular, this allows one to calculate the latter tensor in numerical simulations when the external efforts are in evidence.

Such equality doesn't hold true anymore in dynamic situations, in what case it must be replaced by

$$
\text { int.mom(S) + tens.mom. (Ext.efforts of } \mathrm{S})=\int_{\mathrm{S}} \boldsymbol{x} \otimes \boldsymbol{\gamma}(\boldsymbol{x}) d \mu(\boldsymbol{x}),
$$

where $\gamma$ is the vector field of accelerations and $d \mu$ the mass measure defined on the system.

In Sec. 4 is expanded the contribution to the above integral of a member of the system which would consist of a rigid body. This special material behaviour makes that the acceleration field of the body is completely determined by the external efforts it experiences. In fact, this vector field lets itself be expressed algebraically from the following data: the acceleration vector of some reference point of the body, in practice its mass-center, the spin vector $\boldsymbol{\omega}$ and its time-derivative $\dot{\omega}$. Now, the acceleration of the mass center times the mass equals the resultant vector of 
external efforts, while Euler's equations relate $\dot{\boldsymbol{\omega}}$ to the moment vector of these efforts. In order to derive manageable formulas, one uses orthonormal axes directed along principal directions of the body inertia tensor.

A large set of problems of Granular Mechanics concern dense granular materials in slow motion, so that, macroscopically, the evolution seems quasistatic, i.e. the accelerations observed at the macroscopic scale are estimated to be so small that the "inertia forces" should be negligible with regard to the proper forces involved. However, things may not be so simple at the micromechanical scale. When watching experiments on the slow deformation of a dense granular material, one is used to hear a crackling noise which, in most experimental settings, cannot be ascribed to grain crushing. The time-recording of boundary force measurements, as well as the numerical simulations made by several authors confirm that, in general, evolutions are rather agitated. They involve a succession of crises or "microseisms" which cannot be viewed as quasistatic processes [30, 31, 40, 42]. In two-dimensional experiments with Schneebeli materials (i.e. assemblies of cylindrical rods or prisms of equal lengths, stacked parallel and observed from the axial direction), however slow the overall deformation of the sample is, brutal rearrangements are visible, triggered by sudden local slips.

These brutal episodes are liable to involve, in addition to permanent contacts, some grain collisions. If grains are modelled as rigid bodies collisions require to express interactions non only through contact forces but also through contact percussions. Recall that if a collision affects an element of a cluster of contacting rigid bodies, percussions should be expected at all contact points, not only at the collision locus.

All what precedes was based on the consideration of efforts, in particular contact forces. But, at the instant $t_{c}$ of a collision contact forces may be said to become infinite, making the previous balances meaningless. A balance of percussions should be drawn instead, yielding at the place of $(1)$

$$
\text { tens.mom(Int.percussions })+ \text { tens.mom.(Ext.percussions })=\int_{\mathbf{S}} \boldsymbol{x} \otimes[\boldsymbol{v}(\boldsymbol{x})] d \mu(\boldsymbol{x}),
$$

where $[\boldsymbol{v}(\boldsymbol{x})]$ denotes the time-jump of the velocity vector at point $\boldsymbol{x}$, i.e. the time-limit of this quantity on the right of $t_{c}$ minus its time-limit on the left.

Actually in practice, never a balance pertains to a strict instant but to a certain time-average around this instant. The same is true in time-stepping computations.******

\subsection{Pertinence}

What precedes might induce one to state as a general definition that, for an arbitrary mechanical system $\mathrm{S}$ occupying a domain $\mathrm{D}$, the average stress is the tensor -int.mom(S)/vol(D). Actually, in the rest of this paper, the word "stress" will be used with caution. This is to prevent misunderstandings such as in particular the recurring ones which arise in discussions about the possible lack of symmetry of the stress tensor.*******

\section{Efforts and Virtual Power}

\subsection{Resultant and Moment of an Effort}

According to the formalization of Classical Mechanics in terms of virtual powers (see e.g. [17]), any effort - this is not necessarily a force in the traditional sense - experienced by a material system is defined by the expression of the power (also called "rate of working" [20]) that this effort would develop if the system elements were affected by a class of imagined velocity fields, called virtual velocities or test fields. 
The rule of the game is that this class of vector fields constitutes a linear space and that the real-valued functional "power" is linear on this space. In order to make this formalization able to communicate with Schwartz' theory of distributions, it is assumed that the power is defined at least if the test field $\varphi$ belongs to the function space $\mathcal{C}^{\infty}$ of the indefinitely differentiable vector fields. Accordingly, if $\mathcal{E}$ is an effort, the standard writing $\langle\mathcal{E}, \varphi\rangle$ is used to denote the power of $\mathcal{E}$ for the virtual velocity field $\varphi$.

All the mechanical systems considered in this paper are supposed to occupy bounded portions of space.

In such a framework, take in particular as $\varphi$ the constant vector field with value $\boldsymbol{a}$ everywhere. Since the power $\langle\mathcal{E}, \boldsymbol{\varphi}\rangle$ of $\mathcal{E}$ depends linearly on $\boldsymbol{\varphi}$, i.e. linearly on $\boldsymbol{a}$, there exist (uniquely) some real numbers $R_{i}$ such that $\langle\mathcal{E}, \varphi\rangle=R_{i} a_{i}$.

For simplicity in all this paper, one agrees to use only orthonormal Cartesian coordinate frames. What precedes thus means that the $R_{i}$ are the components of some vector $\mathbf{R}(\mathcal{E})$ such that $\langle\mathcal{E}, \boldsymbol{\varphi}\rangle$ equals the Euclidean scalar product $\mathbf{R}(\mathcal{E}) . \boldsymbol{a}$. This vector is thus independent of the orthonormal frame chosen for computation and is called the resultant of $\mathcal{E}$.

Secondly, let us take as test field the linear vector field $\varphi$ with components

$$
\varphi_{i}(\boldsymbol{x})=b_{i j} x_{j}
$$

The power $\langle\varepsilon, \varphi\rangle$ must depend linearly on the parameters $b_{i j}$, hence there exist (uniquely) some real numbers $M_{i j}$ such that $\langle\mathcal{E}, \boldsymbol{\varphi}\rangle=M_{i j} b_{j i}$. If the Cartesian frame is rotated about its origin $\boldsymbol{o}$, the numbers $M_{i j}$ and $b_{j i}$ are altered, but the invariance of $\langle\mathcal{E}, \boldsymbol{\varphi}\rangle$ makes that the $M_{i j}$ remain the components of a well defined Euclidean tensor of second rank - denote it by $\mathbf{M}(\varepsilon / \boldsymbol{o})$ - called the moment of degree one of $\mathcal{E}$ about the origin. Equivalently, for every $i$ and $j$, the component $M_{i j}$ of this tensor equals the power $\langle\mathcal{E}, \varphi\rangle$ for the following special choice of the vector field $\varphi$

$$
\varphi_{k}(\boldsymbol{x})=\delta_{j k} \delta_{i l} x_{l} .
$$

The change that $\mathbf{M}(\mathcal{E} / \boldsymbol{o})$ undergoes when the origin $\boldsymbol{o}$ is displaced is readily expressed through the following formula; this change vanishes if $\mathbf{R}(\mathcal{E})=\mathbf{0}$.

$$
\mathbf{M}\left(\mathcal{E} / \boldsymbol{o}^{\prime}\right)=\mathbf{M}(\mathcal{E} / \boldsymbol{o})+\left(\boldsymbol{o}-\boldsymbol{o}^{\prime}\right) \otimes \mathbf{R}(\mathcal{E}) .
$$

By axiom, a collection of efforts constitutes itself an effort with the additivity of powers, hence the additivity of resultants and moments. Such an additivity will be accepted in this paper without entering into any of the discussions about countability which are familiar in Integration Theory.

REMARK 1.- Let us insist on our preference for the concepts of virtual velocity and virtual power rather than virtual displacement and virtual work. The latter concepts generate an equivalent formalism since virtual displacements essentially are "infinitesimal" or "variational" vectors, i.e. they equal the formal products of virtual velocity vectors by some formal time-increment, declared infinitesimal. The calculation rules used when dealing with infinitesimal displacement vectors are the same as those holding for velocities in Kinematics, but we find them less clear in practice.

REMARK 2.- An essential aspect of the virtual power formalism, as it is applied in this paper, is that the invoked test fields are only mathematical objects, defined in the ambiant space even at places devoid of any particle of the investigated material system. This contrasts with the prevalent attitude in literature, where it is usually preferred to attach a virtual velocity vector to each particle of the investigated material system. The virtual velocity field is then a vector function defined on the matter, not in the ambiant space, with the drawback that the set of the material particles may possibly fail to be smooth enough for such concepts as the differentiability 
of a vector field to make sense (this is commonly the case on the boundary of of a threedimensional continuum or in models supported by curves or a surfaces).

In the effective motion of a continuous medium, the "real" velocity field may fail to be continuously differentiable. For instance, the presence of jumps of the real velocity field across some surfaces is a familiar feature which does not prevent us from using smooth test fields in the analysis of the situation. The consideration of the power of some efforts in the effective motion and of the corresponding work as its integral over a time-interval, quantities possibly inserted into thermodynamical relationships, is another question.

That the test fields live only at the mathematical level is not a novelty. In traditional Rational Mechanics, the constraint of maintaining a material point in some surface with prescribed motion is a classical topics. It leads to the consideration of the linear space of test velocities said to be "compatible" with the constraint, namely tangent to the surface in its actual position. Since the surface is moving, the proper velocity of the point during an effective motion does not belong to this linear space.

\subsection{Internal efforts}

An effort is declared exerted (or acted) upon a system but one may also specify a material system from which it emanates. By axiom, both terms obey the intuitive syntax: if $\mathrm{S}^{\prime}$ is a subsystem of $\mathrm{S}$, any effort exerted upon (resp. emanating from) $\mathrm{S}^{\prime}$ is also declared to be exerted upon (resp. to emanate from) S.

In particular, the totality of the efforts exerted upon $\mathrm{S}$ and emanating from $\mathrm{S}$ itself is called the total internal effort of this system; notation $\mathcal{E}_{\text {int }}(\mathrm{S})$. Similarly, the totality of the efforts exerted upon S and emanating from the rest of Universe is called the total external effort of S.

In the virtual power formalism, the following generalization of the action-reaction principle is stated as an axiom of Classical Mechanics:

The total internal effort of a material system $\mathrm{S}$ has zero power whenever the test field $\varphi$ equals the velocity field of a rigid motion, i.e. a vector field of the form $\varphi_{i}(\boldsymbol{x})=a_{i}+b_{i j} x_{j}$ with $b_{j i}=-b_{i j}$ (recall that the Cartesian coordinates in use are orthonormal).

This readily implies that the resultant of this effort is zero and that its tensor moment of degree one equals a symmetric tensor $\mathbf{M}^{\mathrm{int}}(\mathrm{S})$ of rank 2 , independent of the choice of the origin. Let us call this tensor the Internal Moment of S.

EXAMPLE. Let $\mathrm{S}$ consist of a portion of a classical continuous medium filling a bounded domain $\Omega$ of space, with $\boldsymbol{\sigma}$ as Cauchy stress field. Then classically, for every continuously differentiable test field $\varphi$,

$$
\left\langle\varepsilon_{\text {int }}(\mathrm{S}), \varphi\right\rangle=-\int_{\Omega} \varphi_{i, j} \sigma_{j i} d \Omega .
$$

This actually constitutes the very definition of the Cauchy stress in a synthetic construction of Continuum Mechanics [17].

Here we comply with the common usage in Mechanical Engineering and in general Continuum Mechanics of counting a stress as positive when it is directed as a traction. In Geomechanics and Civil Engineering the reverse convention is generally applied, requiring to remove the minus sign from the right-hand side of (6).

If one takes as $\varphi$ the same linear vector field as in (3) one has $\varphi_{i, j}=b_{i j}$, thus

$$
M_{i j}^{\mathrm{int}}(\mathrm{S})=-\int_{\Omega} \sigma_{i j} d \omega .
$$

In other words, for such a portion of classical continuous medium, the volume average of the tensor field $\boldsymbol{\sigma}$ equals $-\mathbf{M}^{\text {int }}(\mathrm{S}) / \operatorname{vol}(\Omega)$. 


\section{$2.3 \quad$ Forces}

In familiar situations, efforts merely are forces distributed in the bounded region of space that the concerned system occupies. Such a force repartition $\mathcal{F}$ is mathematically formalized as a measure, say $d \boldsymbol{F}$, with values in the 3 -dimensional vectors. This means that its power makes sense not only for $\varphi \in \mathfrak{C}^{\infty}$ but that the expression of this power may be extended (at least) to any $\boldsymbol{\varphi}$ in the space $\mathbf{e}^{0}$ of the continuous vector fields and is expressed in the form

$$
\langle\mathcal{F}, \boldsymbol{\varphi}\rangle=\int \boldsymbol{\varphi}(\boldsymbol{x}) \cdot d \boldsymbol{F}(\boldsymbol{x})=\int \varphi_{k}(\boldsymbol{x}) d F_{k}(\boldsymbol{x}) ;
$$

the real measures $d F_{k}$ may be called the components of the vector measure $d \boldsymbol{F}$ relative to the coordinate frame in use. One says that $\mathcal{F}$ is an effort of order 0.

This formalism accomodates in particular the case of a discrete collection of forces $f^{\alpha}$ exerted at isolated points $\boldsymbol{x}^{\alpha}$. Each of these forces, said to make an atom (in other words, a vector-weighted Dirac) of the measure $d \boldsymbol{F}$, contributes in the integral (8) by a term of the form $\varphi\left(x^{\alpha}\right) \cdot f^{\alpha}$

By taking $\varphi$ as in (4) one finds that the moment of degree 1 of the discrete collection $\mathcal{F}$ about the origin equals the tensor with components

$$
M_{i j}=\int x_{i} d F_{j}
$$

so that, in coordinate-free notation, one may write

$$
\mathbf{M}(\mathcal{F} / \boldsymbol{o})=\int \boldsymbol{x} \otimes d \boldsymbol{F}(\boldsymbol{x}) .
$$

\subsection{Efforts of order greater than zero}

Let $p$ be a positive integer. In accordance with the usual terminology of the Theory of Distributions, when the effort $\mathcal{E}$ is a vector distribution of order $p$, its power $\langle\mathcal{E}, \boldsymbol{\varphi}\rangle$ makes sense not only for $\varphi \in \mathfrak{C}^{\infty}$ but (at least) for $\varphi$ in the space $\mathfrak{C}^{p}$ of the vector fields which are continuously differentiable up to order $p$.

In view of (6), the total internal effort of a classical continuous medium is of order 1.

Another example of effort of order 1 is provided by a point couple, more appropriately called a point torque, a concept traditionally used in order to conciliate the assumption of two strictly rigid bodies of rotond shapes rolling on each other through a single contact point, with calculations involving some resistance to rolling (see e.g. [25]). Such an effort, say $\mathcal{T}$, is depicted by the point $\boldsymbol{c}$ at which it is localized and by some moment vector $\boldsymbol{m}$. By definition, the power $\langle\mathcal{T}, \varphi\rangle$ corresponding to a test field $\varphi \in \mathcal{C}^{1}$ equals the Euclidean scalar product of $\boldsymbol{m}$ by the

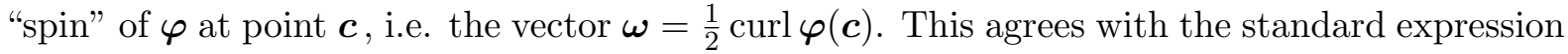
of the power of a couple exerted upon a rigid body, when $\varphi$ equals the velocity field of this body, namely an affine vector field with skew-symmetric matrix. Clearly $\langle\mathcal{T}, \varphi\rangle$ vanishes if $\varphi$ is constant throughout space; therefore $\mathcal{T}$ has zero resultant vector, implying that its vector moment, as well as its tensor moment, do not depend on the choice of an origin.

Here by using the "curl" operator, one assumes that the three-dimensional space is equipped with a physically inessential orientation; if $\varepsilon$ denotes the corresponding orientation tensor, the expression of $\langle\mathcal{T}, \boldsymbol{\varphi}\rangle$ writes down

$$
\boldsymbol{\omega} \cdot \boldsymbol{m}=\frac{1}{2} \varepsilon_{i j k} \varphi_{k, j} m_{i} .
$$

If one takes as $\varphi$ the same linear vector field as in (3), this expression becomes $M_{j k} b_{k j}$, with $M_{j k}=\frac{1}{2} \varepsilon_{i j k} m_{i}$, an antisymmetric tensor. Such is the contribution of the point torque to 
the total tensor moment of the external efforts that the concerned body experiences. Due to the other terms involved, in case of equilibrium, this does not prevent the total from being a symmetric tensor.

Incidentally, observe that the use of the concept of point torque is motivated by the fact that, in the statics or the dynamics of a rigid body, the vector moment $\boldsymbol{m}$ or, equivalently, the associated antisymmetric moment tensor, are all what is needed. Actually the information localized at point $\boldsymbol{c}$ should convey a macroscopic summary of some distribution of contact forces taking place in some very small contact area. Because the surface density of this force distribution takes large and inhomogeneous values, its tensor moment about $\boldsymbol{c}$ turns out to be nonnegligible but then it has no reason for being antisymmetric: the effort of order 1 one localizes at point $\boldsymbol{c}$ is more generally to be viewed as a "multiforce" or "stress multipole" [20].

\subsection{Contact actions}

Let $\mathrm{S}_{1}, \mathrm{~S}_{2}$ denote two materially distinct systems. Among the efforts that $\mathrm{S}_{1}$ may exert upon $\mathrm{S}_{2}$, some are possibly qualified as contact efforts. By this, one means that these efforts are supported by the intersection $\mathrm{K}$ of the boundaries of the parts of space that $\mathrm{S}_{1}$ and $\mathrm{S}_{2}$ occupy (as before, we consider only bounded systems, so that $\mathrm{K}$ is compact). "Supported" here is understood in the mathematical standard sense, that the said efforts have zero power for any test field $\varphi$ which vanishes throughout an open set containing $\mathrm{K}$.

Suppose that $\mathrm{S}$ equals a collection of subsystems $\mathrm{S}_{1}, \mathrm{~S}_{2}, \ldots$ which interact only through contact actions, possibly adhesive.

The syntax of "internal" and "external", as formulated in the foregoing, entails that the internal efforts of S consist of the respective internal efforts of the subsystems and of the mutual efforts that these subsystems may exert upon each other. Since the efforts exerted by $\mathrm{S}_{1}$ upon $\mathrm{S}_{2}$ and those exerted by $\mathrm{S}_{2}$ upon $\mathrm{S}_{1}$ are contact actions (possibly involving torques of resistance to rolling as precedingly described), they are located at the same points of space and, by the action-reaction principle, have opposite values, i.e. opposite powers for any test field (the latter being always continuous). Therefore, the total contribution of these mutual efforts in the internal moment of S vanish, leaving

$$
\mathbf{M}^{\mathrm{int}}(\mathrm{S})=\mathbf{M}^{\mathrm{int}}\left(\mathrm{S}_{1}\right)+\mathbf{M}^{\mathrm{int}}\left(\mathrm{S}_{2}\right)+\ldots
$$

In short, by assigning to every finite collection of subsystems of $\mathrm{S}$ the corresponding internal moments, one defines a finitely additive tensor-valued measure.

No assumption of equilibrium is made here.

The occurence of an integral in (7) makes additivity evident in the special case of a classical continuous medium and shows that the negative of the Cauchy stress field is nothing else than the density function of the internal moment measure relatively to the volume (i.e. Lebesgue) measure. As already observed, the same equation expresses that the volume average of the Cauchy stress field over $\Omega$ equals $-\mathbf{M}^{\text {int }} / \operatorname{vol}(\Omega)$.

Therefore, if a material system $\mathrm{S}$ is viewed as "wrapped" in a bounded domain $\Omega$, it is natural to say that the tensor $-\mathbf{M}^{\text {int }}(\mathrm{S}) / \operatorname{vol}(\Omega)$ constitutes the average stress of $\mathrm{S}$. The uncertainty in defining such a wrapping domain has little consequence if $\mathrm{S}$ is, for instance, a portion of granular material sufficiently dense and comprising sufficiently many grains. It is only at this stage that some assumption of "large number" has to be made. Under such an assumption, it will be numerically checked on the example of Sec.****** that the tensor calculated in that way does possess, up to sampling uncertainty, the main property expected of the Cauchy stress, namely it allows one to predict how the total force transmitted across an ideal cut depends on the direction of this cut. 
The above construction, based on the Virtual Power formalism, has the advantage of possible extension to many other models of continuous media. For instance the internal moment measure of a string or a chain admits a density function relatively to the arc-length measure. The visualisation of force chains in the granular sample of Sec.?? through the internal moments of individual grains suggests that the construction of models of granular media could possibly rest on formulations in which the internal moment measure would not be entirely represented by a density with respect to volume, but comprises tensor measures supported by a network of lines.

\subsection{Interaction bridges}

The additivity property stated in (11) is valid for collections of bodies which interact by contact only. The present theory thus seems to take us very far from the conceptions of Cauchy and his followers [29] whose introduction of stress was based on some underlying microscopic model of crystalline matter consisting of ideally punctual atoms which exert distance-dependent actions on each other.

As for the current Granular Mechanics, the numerical modelling of unsaturated soils commonly rests on a rough way of accounting for the presence of water occupying a small fraction of the space between grains. In three-dimensional situations, this space is usually connected so that air remains free to circulate. Water is then assumed localized in "capillary bridges" materialising an interaction of neighbouring grains without any contact between these rigid bodies. The net effect of surface tension on the free boundary of such liquid bridges is commonly schematized as an attractive force that the two concerned grains would exert upon each other. This action does not consist of mutual contact forces anymore, therefore makes (11) invalid. A protocol to restore additivity is to consider each bridge as an element of the system.

Under the above schematization, a capillary bridge is represented as a massless line segment L connecting a particle $\boldsymbol{b}$ of body B with a particle $\boldsymbol{b}^{\prime}$ of body $\mathrm{B}^{\prime}$. Let L exert a force $\boldsymbol{f}$ on particle $\boldsymbol{b}$ and a force $\boldsymbol{f}^{\prime}$ on particle $\boldsymbol{b}^{\prime}$. By the action-reaction principle, the external forces experienced by L equal the oppposite of the above and, since L is massless, they have the line $\boldsymbol{b} \boldsymbol{b}^{\prime}$ as common support with $\boldsymbol{f}^{\prime}=-\boldsymbol{f}$. The internal moment of L thus equals

$$
\mathbf{M}^{\mathrm{int}}(\mathrm{L})=\boldsymbol{b} \otimes \boldsymbol{f}+\boldsymbol{b}^{\prime} \otimes \boldsymbol{f}^{\prime}=\left(\boldsymbol{b}-\boldsymbol{b}^{\prime}\right) \otimes \boldsymbol{f}
$$

a degenerate symmetric tensor, because $\boldsymbol{f}$ and $\boldsymbol{b}-\boldsymbol{b}^{\prime}$ have the same direction.

If the internal moment of an unsaturated granular sample is evaluated, terms of this form have to be added to the internal moments of grains .

For a collection of distant punctual particles $\boldsymbol{x}_{i}, i=1,2, \ldots, n$, like those considered by Cauchy, let us denote by $\boldsymbol{f}_{i j}$ the force exerted at a distance by $\boldsymbol{x}_{j}$ upon $\boldsymbol{x}_{i}$ (by action-reaction, $\boldsymbol{f}_{j i}=-\boldsymbol{f}_{i j}$ and the direction is that of $\boldsymbol{x}_{i} \boldsymbol{x}_{j}$; by convention $\boldsymbol{f}_{i i}=0$ ). In this case, only bridges contribute in the internal moment of the whole, namely

$$
\mathbf{M}^{\mathrm{int}}=\sum_{i, j} \boldsymbol{x}_{i} \otimes \boldsymbol{f}_{i j}=\sum_{i<j}\left(\boldsymbol{x}_{i}-\boldsymbol{x}_{j}\right) \otimes \boldsymbol{f}_{i j} .
$$

\section{Equilibrium situations}

\subsection{Calculating the internal moment}

No hypothesis was made in the foregoing about the possible motion of the considered mechanical systems. The present section is devoted to the special case of equilibrium relative to a Galilean reference frame. Then the Principle of Virtual Power states that the total power of all the efforts it experiences, namely the internal and the external ones, equals zero whatever is the test field $\varphi$. 
Taking in particular $\varphi$ as in (3), one obtains that if the system $\mathrm{S}$ is in equilibrium,

$$
\mathbf{M}^{\text {int }}(\mathrm{S})=- \text { tens.mom.(Ext.efforts). }
$$

EXAMPLE. Let us consider as before a portion of a classical three-dimensional continuous medium, occupying some bounded domain $\Omega$ with smooth boundary $\partial \Omega$. Assume this portion in equilibrium under peripheral forces, with density function $\boldsymbol{T}$ relative to the area measure $d(\partial \Omega)$ and possibly also some distant external forces distributed with density $\boldsymbol{f}^{\text {dist }}$ relative to the volume measure $d \Omega$.

In view of (7), the equality of $-\mathbf{M}^{\text {int }}$ to the tensor moment of external efforts becomes

$$
\int_{\Omega} \boldsymbol{\sigma}(\boldsymbol{x}) d \Omega=\int_{\partial \Omega} \boldsymbol{x} \otimes \boldsymbol{T}(\boldsymbol{x}) d(\partial \Omega)+\int_{\Omega} \boldsymbol{x} \otimes \boldsymbol{f}^{\text {dist }}(\boldsymbol{x}) d \Omega .
$$

Under the assumption that $\boldsymbol{\sigma}$ is smooth enough, one classically establishes this equality directly, starting from the boundary condition $\boldsymbol{T}=\boldsymbol{\sigma} . \boldsymbol{n}$ which holds on $\partial \Omega$ and from the equation of equilibrium $\operatorname{div} \boldsymbol{\sigma}+\boldsymbol{f}^{\text {dist }}=0$ which holds throughout $\Omega$. Apply to both members of the latter equation the tensor multiplication $\boldsymbol{x} \otimes$, integrate over $\Omega$ and observe that $x_{k}\left(\sigma_{j i, j}+f_{i}^{\text {dist }}\right)=$ $\left(x_{k} \sigma_{j i}\right)_{j}-\delta_{k j} \sigma_{j i}+x_{k} f_{i}^{\text {dist }}$. The Gauss-Ostrogradsky integral formula then yields (14). According to [21], this result may be traced back to C. Chree [7].

Incidentally observe that the right-hand side of (14) is a priori independent of the choice of the origin and symmetric because the assumption of equilibrium classically implies that the resultant of the external efforts vanish, as well as their skew moment, i.e. the antisymmetric part of their tensor moment.

If $\mathrm{S}$ consists of a collection of grains which interact only by contact, the additivity property (11) allows one to calculate the internal moment of each individual grain separately before totalizing the whole. The origin may be chosen arbitrarily for each grain. Taking it at the grain mass center, a point usually at hand in numerical simulations, makes a trick to avoid calculating the contribution of a possible uniform gravity field (automatically taken into account if equilibrium is asserted).

The advantages of this grain-by-grain calculation should not make one forget another possibility. In view of equilibrium, the internal moment of S equals the negative of the tensor moment of the external forces that $\mathrm{S}$ undergoes. The latter comprise the contact forces that the members of S may experience from foreign grains or from confining boundaries but not the contact forces between members of S. If a uniform gravity field is present, calculating its contribution in the moment of external forces requires to determine the mass center of S.

Comparing the results of both modes of calculation provides a checksum test of the numerical quality. The difference allows one to estimate the precision at which the equilibrium conditions of all grains in $\mathrm{S}$ are fullfilled.

\subsection{Saturated soil}

By way of an exception to the rest of this paper, assume in this paragraph that the space between grains is filled with some incompressible homogeneous fluid with density $\rho$. In usual experiments with "dry" granulates, this fluid is air, whose density may in fact be viewed as a constant in the considered portion of space. The following is rather aimed at the case of a granular soil with interstitial space entirely occupied by water. Everything is assumed in equilibrium, so that the possible viscosity of the fluid plays no part. Gravity with intensity $g>0$ is taken into account. Denoting by $z(\boldsymbol{x})$ the altitude of a point $\boldsymbol{x}$, the equation of Hydrostatics allows one to determine the pressure at some point $\boldsymbol{x}$ of the fluid by $p=\varpi(\boldsymbol{x})$, where $\varpi$ denotes the function defined everywhere by

$$
\varpi(\boldsymbol{x})=h-\rho g z(\boldsymbol{x}) .
$$


Here $h$ is a constant in each connected component of the fluid domain. As usual in threedimensional situations, it is assumed that the fluid domain consists of a single connected component.

Denoting by $\Omega$ a probe domain, let us begin with the special case where this domain is entirely filled with fluid, without any grain. This makes a portion of classical continuous medium with Cauchy stress tensor $\sigma_{i j}=-p \delta_{i j}$. In view of (7), its internal moment has components

$$
-\int_{\Omega} \sigma_{i j} d v=\delta_{i j} \int_{\Omega} \varpi d v
$$

Since $\varpi$ is an affine function of the coordinates, its integral over $\Omega$ equals $\operatorname{vol}(\Omega) \varpi\left(\boldsymbol{c}_{\Omega}\right)$, where $\boldsymbol{c}_{\Omega}$ denotes the geometric center of $\Omega$, i.e. the mass-center of a material of uniform density imagined to fill this domain.

Coming now to the case where $\Omega$ contains also a collection of possibly contacting grains which leave only as fluid domain the complementary subset $\Phi$, one relies on the additivity property (11) to calculate the internal moment of the whole. For every grain, say B, one uses (13) to express its internal moment from the external forces it undergoes. These are contact forces emanating from other grains or from confining boundaries, as they may be computed in a numerical simulation and in addition the pressure forces from the fluid. Assume first that B is entirely immersed, i.e. the points at which contact forces are applied to it make a part of $\partial \mathrm{B}$ with negligible area. At every point $\boldsymbol{x}$ of $\partial \mathrm{B}$, denote by $\boldsymbol{n}(\boldsymbol{x})$ the outward normal unit. The tensor moment of the pressure forces experienced by $\mathrm{B}$ has components

$$
-\int_{\partial \mathrm{B}} x_{i} \varpi n_{j} d(\partial \mathrm{B})=-\int_{\mathrm{B}}\left(x_{i} \varpi\right)_{, j} d \mathrm{~B}=-\delta_{i j} \int_{\mathrm{B}} \varpi d \mathrm{~B}-\int_{\mathrm{B}} x_{i} \varpi, j d \mathrm{~B} .
$$

Now $\varpi, j=-\rho g \zeta_{j}$, with $\boldsymbol{\zeta}$ denoting the upward vertical unit vector and $\int_{B} \varpi d \mathrm{~B}=\operatorname{vol}(\mathrm{B}) \varpi\left(\boldsymbol{c}_{\mathrm{B}}\right)$. The above components thus equal

$$
-\operatorname{vol}(\mathrm{B}) \varpi\left(\boldsymbol{c}_{\mathrm{B}}\right) \delta_{i j}+\operatorname{vol}(\mathrm{B}) \rho g c_{i \mathrm{~B}} \zeta_{j}
$$

This yields as the internal moment of the whole contents of $\Omega$ the tensor with components:

$$
\left(\operatorname{vol}(\Phi) \varpi\left(\boldsymbol{c}_{\Phi}\right)+\sum_{\mathrm{B}} \operatorname{vol}(\mathrm{B}) \varpi\left(\boldsymbol{c}_{\mathrm{B}}\right)\right) \delta_{i j}-\left(\sum_{\mathrm{B}} \operatorname{vol}(\mathrm{B}) \rho g c_{i \mathrm{~B}}\right) \zeta_{j}-\sum_{\mathrm{B}} M_{i j}^{\mathrm{ext}}(\mathrm{B})
$$

The term in $\delta_{i j}$ simply equals $\operatorname{vol}(\Omega) \varpi\left(\boldsymbol{c}_{\Omega}\right) \delta_{i j}$, the result when $\Omega$ contains no grain. The term $-\sum_{\mathrm{B}} M_{i j}^{\text {ext }}(\mathrm{B})$ would be the result of the standard calculation of the internal moment of the grain collection, taking into account the external contact forces and the weights of grains (whose mass distribution does not need to be homogeneous), but ignoring the fluid pressure. The intermediate term may be viewed as an Archimedian correction, accounting for the presence of the fluid: $-\operatorname{vol}(\mathrm{B}) \rho g c_{i \mathrm{~B}} \zeta_{j}$ are the components of the tensor moment (about the same origin as that used in calculating the other terms) of the gravity forces experienced by a fictitious homogeneous body with density $\rho$ occupying the place of B.

It is for simplicity that the contact zones of the grains have been assumed of zero area. Actually, if grain B touches grain B', with outward normal unit $\boldsymbol{n}^{\prime}(\boldsymbol{x})=-\boldsymbol{n}(\boldsymbol{x})$, along a contact zone of nonzero area, one does not change the above balance by introducing the force distribution $-\varpi(\boldsymbol{x}) \boldsymbol{n}(\boldsymbol{x})$ on the corresponding part of $\partial \mathrm{B}$ and the force distribution $-\varpi(\boldsymbol{x}) \boldsymbol{n}^{\prime}(\boldsymbol{x})$ on the corresponding part of $\partial \mathrm{B}$ '. Similarly, if grain B touches an obstacle (i.e. an external grain or a material boundary) along a zone $\mathrm{C}$ of nonzero area, one does not change balances by imagining that $\mathrm{C}$ is occupied by a layer of fluid which exerts the force distribution $-\varpi(\boldsymbol{x}) \boldsymbol{n}(\boldsymbol{x})$ on the corresponding part of $\partial \mathrm{B}$ an the opposite force distribution on the obstacle. 


\subsection{Piece of string}

The model of an infinitely thin string is a chain of particles labelled by a parameter $\lambda$ ranging in a real interval $\Lambda$, assumed compact for brevity. A placement of the string into the Euclidean space $\mathbb{E}$ of a reference frame is a mapping $\boldsymbol{P}: \Lambda \rightarrow \mathbb{E}$, that we suppose continuous with bounded variation (in other words, this is a "rectifiable curve"). As for the mechanical behaviour, it is put in this Parag. that the string is perfectly flexible in the following sense: for every $\bar{\lambda}$ in the interior of $\Lambda$, the part $\lambda>\bar{\lambda}$ of the string exerts upon the part $\lambda<\bar{\lambda}$ a simple force $\Theta(\bar{\lambda})$ localized at point $\boldsymbol{P}(\bar{\lambda})$ (but no point torque as there would exist in the case of a string able to oppose some reluctance to flexion).

Only equilibrium conditions are investigated in what follows. In the traditional approach to this question, $\boldsymbol{P}$ is assumed continuously differentiable, making of the placement a smooth curve. The string is supposed submitted to distributed external forces $d \boldsymbol{F}=\boldsymbol{F}_{s}{ }^{\prime}(\lambda) d s$ with $d s=\left\|\boldsymbol{P}^{\prime}(\lambda)\right\| d \lambda$. By writing that for every subinterval of $\Lambda$, the resultant and resultant moment of the external forces that the corresponding piece of the string undergoes, one classically obtains

- The vector $\Theta(\lambda)$ is tangent to the curve at point $\lambda$, i.e. there exists a real number $\Theta(\lambda)$, called the tension of the string, such that $\boldsymbol{\Theta}(\lambda)=\Theta(\lambda) \boldsymbol{T}(\lambda)$ where $\boldsymbol{T}(\lambda)=\boldsymbol{P}^{\prime}(\lambda) /\left\|\boldsymbol{P}^{\prime}(\lambda)\right\|$ denotes the tangent unit vector in the direction of increasing $\lambda$.

- The differential equation

$$
d(\Theta \boldsymbol{T})+d \boldsymbol{F}=0
$$

is verified at every point of the string.

Even if some conditions at the endpoints of the string are added, this 3-component differential equation is not enough to determine the three components of $\boldsymbol{P}$ and the real number $\Theta$ as functions of $\lambda$. Some phenomenological information about the mechanical properties of the string should be provided in the form of a "constitutive law" which connects $\Theta$ with some geometric properties of the placement. An extreme case is that of an inextensible string. Geometrically, inextensibility means that, whatever the placement is, $\left\|\boldsymbol{P}^{\prime}(\lambda)\right\|$ equals a given function of $\lambda$. The unknown function $\Theta(\lambda)$ then plays the part of the "multiplier" or "reaction" associated with this mechanical constraint.

Since the string is assumed in equilibrium, (13) may be used to assess, for every virtual velocity field $\varphi$, the power of the internal effort of a portion $\left[\lambda_{0}, \lambda_{1}\right]$ of the string, namely

$$
\begin{aligned}
\mathcal{P}^{\text {int }}(\boldsymbol{\varphi},[ & {\left.\left[\lambda_{0}, \lambda_{1}\right]\right)=-\mathcal{P}^{\operatorname{ext}}\left(\boldsymbol{\varphi},\left[\lambda_{0}, \lambda_{1}\right]\right) } \\
& =-\int_{\left[\lambda_{0}, \lambda_{1}\right]} \boldsymbol{\varphi}(\boldsymbol{P}) \cdot d \boldsymbol{F}-\boldsymbol{\varphi}\left(\boldsymbol{P}\left(\lambda_{1}\right)\right) . \Theta\left(\lambda_{1}\right) \boldsymbol{T}\left(\lambda_{1}\right)+\boldsymbol{\varphi}\left(\boldsymbol{P}\left(\lambda_{0}\right)\right) \cdot \Theta\left(\lambda_{0}\right) \boldsymbol{T}\left(\lambda_{0}\right) .
\end{aligned}
$$

If one extracts from (16) the expression of $d \boldsymbol{F}$, the integral on the right-hand side becomes

$$
\int_{\left[\lambda_{0}, \lambda_{1}\right]} \boldsymbol{\varphi}(\boldsymbol{P}) \cdot d(\Theta \boldsymbol{T})=\int_{\left[\lambda_{0}, \lambda_{1}\right]} d(\boldsymbol{\varphi}(\boldsymbol{P}) \cdot \Theta \boldsymbol{T})-\int_{\left[\lambda_{0}, \lambda_{1}\right]} \Theta \boldsymbol{T} \cdot d(\boldsymbol{\varphi}(\boldsymbol{P})) .
$$

Now, denoting by $S$ the arc length directed according to increasing $\lambda$, one has

$$
\boldsymbol{T} . d(\boldsymbol{\varphi}(\boldsymbol{P}))=T_{i} \varphi_{i, j} T_{j} d S=T_{i} T_{j}(\operatorname{def} \boldsymbol{\varphi})_{i j} d S
$$

where $(\operatorname{def} \varphi)_{i j}=\left(\varphi_{i, j}+\varphi_{j, i}\right) / 2$ are the components of the deformation rate tensor of a continuous medium admitting $\varphi$ as Eulerian velocity field. Classically, $T_{i} T_{j}(\operatorname{def} \boldsymbol{\varphi})_{i j}$ expresses the local stretching rate of a chain of particles of this medium which would possess $\boldsymbol{T}$ as tangent 
unit vector. Denoting by $E$ the value of this stretching rate at the generic point of the string, one writes (17) in the form

$$
\mathcal{P}^{\operatorname{int}}\left(\varphi,\left[\lambda_{0}, \lambda_{1}\right]\right)=-\int_{\left[\lambda_{0}, \lambda_{1}\right]} \Theta E d S
$$

If, in particular, one takes as $\varphi$ an affine vector field, this yields the internal moment of the piece of string

$$
\mathbf{M}^{\text {int }}\left[\lambda_{0}, \lambda_{1}\right]=-\int_{\left[\lambda_{0}, \lambda_{1}\right]} \Theta \boldsymbol{T} \otimes \boldsymbol{T} d S .
$$

The standard theory of perfectly flexible strings, which generates the differential equation (16), rests on the assumption of continuous differentiability for the placement mapping $\boldsymbol{P}$. A more general setting, with $\boldsymbol{P}$ only assumed continuous with bounded variation, will have the advantage of accepting, for instance, that the string present a corner point at its contact with an obstacle edge. Bounded variation implies the existence of a measure on the interval $\Lambda$ with values in the linear space $\mathbf{E}$ of the vectors of $\mathbb{E}$, called the Stieltjes measure or "differential measure" of $\boldsymbol{P}$, denoted here by $d \boldsymbol{P}$. If $\mathbf{E}$ is equipped with the Euclidean norm, one associates with the vector measure $d \boldsymbol{P}$ some nonnegative real measure on $\Lambda$ called its absolute value, denoted by $\|d \boldsymbol{P}\|$. The integral of $\|d \boldsymbol{P}\|$ on any subinterval is by definition the length of the corresponding arc of the curve, a definition consistent with the meaning of length in the case of a smooth curve.

The decisive move now is to introduce the images of the above measures under the continuous mapping $\boldsymbol{P}: \Lambda \rightarrow \mathbb{E}$. These images are measures defined in $\mathbb{E}$ instead of $\Lambda$.

For every real test function $\varphi \in \mathfrak{C}^{\infty}$, the real-valued functional

$$
\varphi \mapsto \int_{\Lambda} \varphi(\boldsymbol{P}(\lambda))\|d \boldsymbol{P}(\lambda)\|
$$

is readily found to possess the continuity property required for constituting a real-valued measure on $\mathbb{E}$. Let us denote by $d s$ this measure, so that the above real value is written $\int \varphi d s$. This symbol is found to make sense also for $\varphi \in \mathfrak{C}^{0}$ and even for the $d s$-specific larger class of functions said $d s$-integrable. The support of the measure $d s$ equals the position of the string, i.e. the subset $\boldsymbol{P}(\Lambda)$ of $\mathbb{E}$; in fact the above expression obviously equals zero for every test function $\varphi$ which vanishes in an open subset containing $\boldsymbol{P}(\Lambda)$.

Similarly, for every $\varphi \in \mathfrak{C}^{\infty}$, the functional

$$
\varphi \mapsto \int_{\Lambda} \varphi(\boldsymbol{P}(\lambda)) d \boldsymbol{P}(\lambda)
$$

constitutes a $\mathbf{E}$-valued measure on $\mathbb{E}$, noted by $d \boldsymbol{p}$; this is the image of $d \boldsymbol{P}$ under the continuous mapping $\boldsymbol{P}: \Lambda \rightarrow \mathbb{E}$. From the rules for "change of variable" in Integration Theory, it ensues that this measure remains the same if the labelling of the elements of the string is changed through an increasing continuous mapping $\lambda \mapsto \lambda^{\prime}$ In that sense, the vector measure $d \boldsymbol{p}$ appears more intimely associated than $d \boldsymbol{P}$ to the mechanical situation.

Due to standard facts of Integration Theory, $d \boldsymbol{p}$ possesses a density function relative to the nonnegative measure $d s=\|d \boldsymbol{p}\|$; this is a $d s$-integrable function whose values are elements of $\mathbf{E}$ with norm equal to $1 d s$-almost everywhere. Let us denote by $\boldsymbol{\tau}$ this vector function defined $d s$ almost everywhere in $\mathbb{E}$; of course, when $\boldsymbol{P}$ is continuously differentiable, $\boldsymbol{\tau}$ equals, in standard sense, the tangent unit vector to the curve $\boldsymbol{P}(\Lambda)$ in the direction of increasing $\lambda$. Since $\boldsymbol{\tau}$ is only defined $d s$-almost everywhere, the values one attributes to it outside $\boldsymbol{P}(\Lambda)$ are immaterial and it is convenient to figure them out as zero.

Coming now to Kinematics, let us imagine as before that the test vector field $\boldsymbol{\varphi} \in \mathfrak{C}^{\infty}$ is the Eulerian velocity field of a continuous medium which convects the particles of the string. Because this continuous medium presents a very smooth deformation process, one may show 
that, in spite of $\boldsymbol{P}$ possibly beeing nondifferentiable, the time-derivative of the length of a string arc equals the integral of $e d s$ over this arc, with $e=\tau_{i} \tau_{j}(\operatorname{def} \varphi)_{i j}$. The difference from the foregoing is that $d s$ now is a real measure in the space $\mathbb{E}$ and that $\boldsymbol{\tau}$ and $e$ are functions defined $d s$-almost everywhere in this space. For mathematical details about the transport of calculative objects by such an imagined continuous medium, called a carrier, see [34][35].

Thereby we are entering a mechanical formulation in the "Eulerian" style, while the labelling of each particle of the string by some $\lambda$ in $\Lambda$, used in the foregoing, is "Lagrangian" practice.

In the framework of virtual velocities, the definition of the model in view rests on the expression of the power of the internal efforts. As one wants it consistent with the precedings, this expresssion introduces a $d s$-integrable real function $\vartheta$ (defined $d s$-everywhere in $\mathbb{E}$ ) and it is stated that the power of the internal efforts of a portion of the string equals the integral of the real measure $-\theta e d s$ over this portion.

By taking in particular for $\varphi$ an affine vector field, one finds that the internal moment of the portion equals the integral of the tensor measure $-\theta \boldsymbol{\tau} \otimes \boldsymbol{\tau} d s$. One sees here the tensor quantity $\theta \boldsymbol{\tau} \otimes \boldsymbol{\tau}$ playing a role similar to that of $\boldsymbol{\sigma}$ in (7).

Suppose the piece of string in equilibrium under external forces represented by $d \boldsymbol{F}$, an Evalued measure in $\mathbb{E}$. The total virtual power of internal and external forces should vanish for every test field $\varphi$, i.e.

$$
\int \varphi_{i} d F_{i}+\int \vartheta \tau_{i} \tau_{j} \varphi_{i, j} d s=0 .
$$

The definition of the partial derivatives of distributions makes that

$$
\int \vartheta \tau_{i} \tau_{j} \varphi_{i, j} d s=-\left\langle\left(\vartheta \tau_{i} \tau_{j}\right)_{, j}, \varphi_{i}\right\rangle=-\left\langle\operatorname{div}(\vartheta \boldsymbol{\tau} \otimes \boldsymbol{\tau})_{, i}, \varphi_{i}\right\rangle
$$

Therefore (20) becomes

$$
\operatorname{div}(-\vartheta \boldsymbol{\tau} \otimes \boldsymbol{\tau} d s)+d \boldsymbol{F}=0
$$

quite similar to the equation of the statics of a three-dimensional continuous medium. This formal uniformity is to be exploited in $* * *$ below.

As an example of non-differentiability for $\boldsymbol{P}$, let us assume that the curve $\boldsymbol{P}(\Lambda)$ exhibits a corner at some point $\boldsymbol{c}=\boldsymbol{P}\left(\lambda_{c}\right)$, with tangent units $\boldsymbol{\tau}^{-}$and $\boldsymbol{\tau}^{+}$on the left and on the right of $\boldsymbol{c}$ and that $\vartheta$ correspondingly admits limits $\vartheta^{-}$and $\vartheta^{+}$. We are to show that the vector distribution $\operatorname{div}(-\vartheta \boldsymbol{\tau} \otimes \boldsymbol{\tau} d s)$, which actually is a vector measure by virtue of (21), possesses at point $\boldsymbol{c}$ an atom of weight $\vartheta^{+} \boldsymbol{\tau}^{+}-\vartheta^{-} \boldsymbol{\tau}^{-}$. Technically, this makes an example the articulation between Eulerian and Lagrangian calculations.

The $i$-th component of $\operatorname{div}(-\vartheta \boldsymbol{\tau} \otimes \boldsymbol{\tau} d s)$ is the distribution which associates with every test function $\varphi$ the real number

$$
\left\langle\left(-\vartheta \tau_{i} \tau_{j} d s\right)_{j}, \varphi\right\rangle=\left\langle\vartheta \tau_{i} \tau_{j} d s, \varphi, j\right\rangle=\int_{\mathbb{E}} \vartheta \tau_{i} \tau_{j} \varphi_{, j} d s .
$$

\subsection{A glance at the standard approaches to granular stress}

Let $\mathrm{S}$ denote a sample extracted from a granulate in equilibrium. A reasonable idea is that the average stress tensor of this sample should be defined by adapting relation (14) to the discrete distribution of contact forces [11].

Let us call X the world outside $\mathrm{S}$ : a force experienced by a member $\mathrm{B}$ of $\mathrm{S}$ is declared external to $\mathrm{S}$ if it emanates from some member $\mathrm{E}$ of $\mathrm{X}$, in which case we denote by $f^{\mathrm{BE}}$ its vector value. The agent $\mathrm{E}$ may be a grain not belonging to $\mathrm{S}$ or also some external boundary and we call $c^{\mathrm{BE}}$ the position vector of the point of application of the said force. One agrees to extend this formalism so as to include the forces arising from a gravity field constant in the concerned region of space. Actually, the effect of gravity upon body B does not consist in general of a discrete 
collection of forces since it is distributed proportionally to mass, but so long as one is interested only in the tensor moment of this force distribution, it may be replaced by a single force applied to the mass center of $\mathrm{B}$, whose position vector is noted $\boldsymbol{c}^{\mathrm{BG}}$ (symbolically $\mathrm{G}$ stands for gravity, formally viewed as a member of $\mathrm{X}$ ).

By analogy with (14), one is thus induced to put the definition

$$
\text { mean stress }(\mathrm{S}):=\frac{1}{\operatorname{vol}(\mathrm{S})} \sum_{\mathrm{B} \in \mathrm{S}} \sum_{\mathrm{E} \in \mathrm{X}} c^{\mathrm{BE}} \otimes f^{\mathrm{BE}},
$$

where $\operatorname{vol}(\mathrm{S})$ denotes the volume of the region of space covered by the granular sample, including the intersticial space. The uncertainty in identifying this volume is minimized if grains are numerous and closely packed.

The assumption that $\mathrm{S}$ is in equilibrium entails that the expression on the right-hand side does not depend on the origin of position vectors and equals a symmetric tensor.

For simplicity here, contact actions have been assumed to consist of single forces localized at each contact point, without considering any of the "point torques" invoked in Parag. 2.4. If such torques were present among the external efforts undergone by S, their moment tensors should be additionally taken into account on the right-hand side of (22). Due to equilibrium, the total expression would still make a symmetric tensor.

The PAIR BY PAIR CALCULATION.- Inside every grain, say B, a reference point is selected, with position vector $\boldsymbol{r}^{\mathrm{B}}$. Commonly in literature this point is taken at the mass center of $\mathrm{B}$, a choice devoid of mechanical relevance since in the context of Statics, the mass distribution in the grain plays no part. By putting $\boldsymbol{l}^{\mathrm{BE}}=\boldsymbol{c}^{\mathrm{BE}}-\boldsymbol{r}^{\mathrm{B}}$, one gives to (22) the form

$$
\text { mean stress }(\mathrm{S}):=\frac{1}{\operatorname{vol}(\mathrm{S})} \sum_{\mathrm{B} \in \mathrm{S}} \sum_{\mathrm{E} \in \mathrm{X}} \boldsymbol{l}^{\mathrm{BE}} \otimes \boldsymbol{f}^{\mathrm{BE}}+\frac{1}{\operatorname{vol}(\mathrm{S})} \sum_{\mathrm{B} \in \mathrm{S}} \sum_{\mathrm{E} \in \mathrm{X}} \boldsymbol{r}^{\mathrm{B}} \otimes \boldsymbol{f}^{\mathrm{BE}}
$$

The last term is now transformed through a discrete analog to equality (14) in order to generate an expression involving the contact forces that the members of $\mathrm{S}$ exert on each other.

Since every member B of S is a (possibly deformable) body in equilibrium, the sum of all the forces external to itself it experiences equals zero, i.e.

$$
\sum_{\mathrm{E} \in \mathrm{X}} \boldsymbol{f}^{\mathrm{BE}}+\sum_{\mathrm{B}^{\prime} \in \mathrm{S}} \boldsymbol{f}^{\mathrm{BB}}=0
$$

Here $f^{\mathrm{BB}^{\prime}}$ denotes the sum of the contact forces that grain $\mathrm{B}^{\prime}$ exerts upon grain $\mathrm{B}$ with the convention that $\boldsymbol{f}^{\mathrm{BB}^{\prime}}=0$ if $\mathrm{B}$ and $\mathrm{B}^{\prime}$ have no contact (and also if $\mathrm{B}=\mathrm{B}^{\prime}$ ). No assumption is made about grain shapes, hence several contact points may be present for each pair.

Through tensor multiplication by $\boldsymbol{r}^{\mathrm{B}}$, then summation for all B in S, equality (24) implies

$$
\sum_{\mathrm{B} \in \mathrm{S}} \sum_{\mathrm{E} \in \mathrm{X}} \boldsymbol{r}^{\mathrm{B}} \otimes \boldsymbol{f}^{\mathrm{BE}}=-\sum_{\mathrm{B} \in \mathrm{S}} \sum_{\mathrm{B}^{\prime} \in \mathrm{S}} \boldsymbol{r}^{\mathrm{B}} \otimes \boldsymbol{f}^{\mathrm{BB}}
$$

Due to the action-reaction principle, $f^{\mathrm{BB}^{\prime}}=-\boldsymbol{f}^{\mathrm{B}^{\prime} \mathrm{B}}$. In the right-hand side of (25), $\mathrm{B}$ and $\mathrm{B}$ ' are independent summation indices, so the same total is obtained if the names of these indices are exchanged; finally this right-hand side equivalently reads

$$
\frac{1}{2} \sum_{\mathrm{B} \in \mathrm{S}} \sum_{\mathrm{B}^{\prime} \in \mathrm{S}}\left(\boldsymbol{r}^{\mathrm{B}}-\boldsymbol{r}^{\mathrm{B}^{\prime}}\right) \otimes \boldsymbol{f}^{\mathrm{B}^{\prime} \mathrm{B}}=\sum_{\mathrm{B}} \sum_{\mathrm{B}^{\prime} \prec \mathrm{B}} \boldsymbol{l}^{\mathrm{B}^{\prime} \mathrm{B}} \otimes \boldsymbol{f}^{\mathrm{B}^{\prime} \mathrm{B}},
$$

where $\boldsymbol{l}^{\mathrm{B}^{\prime} \mathrm{B}}=\boldsymbol{r}^{\mathrm{B}}-\boldsymbol{r}^{\mathrm{B}^{\prime}}$ is the vector going from the reference point of $\mathrm{B}^{\prime}$ to the reference point of B. Here a strict ordering $\prec$ has been introduced over the collection $\mathrm{S}$ (for instance the ordering 
induced by numbering grains), so as to prevent each term from occuring twice in the summation. Therefore (23) becomes

$$
\text { mean stress }(\mathrm{S}):=\frac{1}{\operatorname{vol}(\mathrm{S})} \sum_{\mathrm{B} \in \mathrm{S}} \sum_{\mathrm{E} \in \mathrm{X}} \boldsymbol{l}^{\mathrm{BE}} \otimes \boldsymbol{f}^{\mathrm{BE}}+\frac{1}{\operatorname{vol}(\mathrm{S})} \sum_{\mathrm{B}} \sum_{\mathrm{B}^{\prime} \prec \mathrm{B}} \boldsymbol{l}^{\mathrm{B}^{\prime} \mathrm{B}} \otimes \boldsymbol{f}^{\mathrm{B}^{\prime} \mathrm{B}} .
$$

The last term involves only contact forces internal to the sample, in contrast with the expression in (22) which only involves forces external to this sample. This last term is frequently used in literature as a definition of the mean stress. As a consequence of neglecting the first term of the right-hand side above, the tensor so defined presents the defects of depending on the choice of the reference points in grains and of exhibiting a lack of symmetry subject to this dependance. It provides only an approximation of the expression (22); an upper bound of the discrepancy may be calculated in terms of the Euclidean norms of tensors and vectors and of the maximal diameter of grains, since $\boldsymbol{c}^{\mathrm{BE}}$ lies in the volume of grain $\mathrm{B}$ as well as, by choice, the reference point $\boldsymbol{r}^{\mathrm{B}}$

$$
\left\|\sum_{\mathrm{B} \in \mathrm{S}} \sum_{\mathrm{E} \in \mathrm{X}} \boldsymbol{l}^{\mathrm{BE}} \otimes \boldsymbol{f}^{\mathrm{BE}}\right\| \leq \max . \operatorname{diam} \times \sum_{\mathrm{B} \in \mathrm{S}} \sum_{\mathrm{E} \in \mathrm{X}}\left\|\boldsymbol{f}^{\mathrm{BE}}\right\| .
$$

REMARK.- The above transformation of (22) into an expression involving the contact forces internal to the sample $\mathrm{S}$ achieves some improvement upon what is usually done in literature, since here several contacts points are accepted in a pair of grains. This is necessary, for instance, in the numerical simulations of polygonal grains. At the cost of more cumbersome notations, it would even be possible to improve generality by considering that, due to deformability, the contact between two grains actually takes place through some parts of their respective surfaces not reduced to points. The corresponding resultant forces, expressed as integrals, would just have to be inserted into (26).

\section{One step into Dynamics}

\subsection{Introducing the acceleration field}

Equation (13) pertains to the special case of equilibrium. If, more generally, the motion of $\mathrm{S}$ relative to some inertial reference frame, under some definite mechanical actions, has been calculated, the synthetic formulation of Classical Mechanics [17] implies that, for every test field $\varphi$, the virtual power of all the efforts, internal as well as external, experienced by the system equals that of the vector measure $\gamma(\boldsymbol{x}) d \mu(\boldsymbol{x})$, where $\gamma$ denotes the acceleration field and $d \mu$ the mass mesure. By using as $\varphi$ the same affine vector field as before, one obtains, for every subsystem s of S, in coordinate-free notation,

$$
\left.\mathbf{M}^{\mathrm{int}}(\mathrm{s})=- \text { tens.mom. (Ext.efforts of } \mathrm{s}\right)+\int_{\mathrm{s}} \boldsymbol{x} \otimes \boldsymbol{\gamma}(\boldsymbol{x}) d \mu(\boldsymbol{x}) .
$$

The total expression on the right-hand side, like $\mathbf{M}^{\text {int }}(\mathrm{s})$ itself, is sure to be independent of the choice of the origin and to constitute a symmetric tensor.

This applies in particular to each member, possibly deformable, of a granular material. As in the equilibrium case, the additivity of $\mathbf{M}^{\text {int }}$ which holds if only contact interactions are present (otherwise, one has to add the internal moments of the interaction bridges) allows one to calculate the contribution of each grain separately by using an ad hoc origin. If, in the exploitation of (28), the origin is chosen at the center of mass of the fragment s, the contribution of the gravity terms in the tensor moment of external efforts vanishes. Gravity has of course be taken into account when calculating the motion. 


\subsection{Rigid bodies}

Let $\mathrm{B}$ denote a rigid body. If it moves relatively to the Galilean frame in use, the application of (28) requires calculating the integral

$$
\int_{\mathrm{B}} \boldsymbol{x} \otimes \boldsymbol{\gamma}(\boldsymbol{x}) d \mu(\boldsymbol{x})
$$

If $\boldsymbol{b}$ denotes a point following B in its motion, the velocity field of this rigid body is expressed at any instant by

$$
\boldsymbol{v}(\boldsymbol{x})=\boldsymbol{v}(\boldsymbol{b})+\boldsymbol{\omega} \times(\boldsymbol{x}-\boldsymbol{b}),
$$

where $\boldsymbol{\omega}$ is the spin vector of B. By time-derivation relative to the same frame, this yields the acceleration field

$$
\begin{aligned}
\gamma(\boldsymbol{x}) & =\gamma(\boldsymbol{b})+\dot{\boldsymbol{\omega}} \times(\boldsymbol{x}-\boldsymbol{b})+\boldsymbol{\omega} \times(\boldsymbol{v}(\boldsymbol{x})-\boldsymbol{v}(\boldsymbol{b})) \\
& =\gamma(\boldsymbol{c})+\dot{\boldsymbol{\omega}} \times(\boldsymbol{x}-\boldsymbol{b})+\boldsymbol{\omega} \times(\boldsymbol{\omega} \times(\boldsymbol{x}-\boldsymbol{b}))
\end{aligned}
$$

where $\dot{\boldsymbol{\omega}}$ denotes the time-derivative of $\boldsymbol{\omega}$ relative to the reference frame. Importantly, $\dot{\boldsymbol{\omega}}$ also equals the time derivative of $\boldsymbol{\omega}$ relative to a frame attached to the rigid body, because the change of frame results in the corrective term $\boldsymbol{\omega} \times \boldsymbol{\omega}$, namely zero.

The integral in (29) thus splits into three terms. First

$$
\int_{\mathrm{B}} \boldsymbol{x} \otimes \boldsymbol{\gamma}(\boldsymbol{b}) d \mu(\boldsymbol{x})=\left(\int_{\mathrm{B}} \boldsymbol{x} d \mu(\boldsymbol{x})\right) \otimes \boldsymbol{\gamma}(\boldsymbol{b})
$$

\section{Introducing the mass center}

- The integral in (30) vanishes if the origin from which the position vector $\boldsymbol{x}$ is evaluated coincides, at the considered instant, with the mass-center of $\mathrm{B}$, an assumption made in all the sequel.

The use of the cross product $x$ in what precedes rests on the choice of some (physically unsignificant) orientation of the three-dimensional space; let us denote by $\varepsilon$ the corresponding orientation tensor. The components $\varepsilon_{i j k}$ vanish except when $\{i, j, k\}$ is a permutation of $\{1,2,3\}$. If the orthonormal coordinates $\left(x_{1}, x_{2}, x_{3}\right)$ are positively ordered with regard to the chosen orientation of space, $\varepsilon_{i j k}$ takes the values +1 or -1 according to the "parity" of the said permutation. Hence

$$
(\boldsymbol{x} \otimes(\dot{\boldsymbol{\omega}} \times \boldsymbol{x}))_{h i}=x_{h} \varepsilon_{i j k} \dot{\omega}_{j} x_{k} .
$$

By integration this yields the second term

$$
T_{h i}^{*}=\left(\int_{\mathrm{B}} \boldsymbol{x} \otimes(\dot{\boldsymbol{\omega}} \times \boldsymbol{x}) d \mu\right)_{h i}=\varepsilon_{i j k} \dot{\omega}_{j} I_{h k}
$$

where $\boldsymbol{I}$ denotes the "planar" inertia tensor $\int_{B} \boldsymbol{x} \otimes \boldsymbol{x} d \mu$ of B (to be distinguished from the "axial" inertia tensor, classically used when expressing from $\boldsymbol{\omega}$ the kinetic energy of B).

Finally, because the properties of $\varepsilon$ entail

$$
(\boldsymbol{\omega} \times(\boldsymbol{\omega} \times \boldsymbol{x}))_{i}=\omega_{j} x_{j} \omega_{i}-\omega_{j} \omega_{j} x_{i}
$$

integration yields the third term

$$
T_{h i}^{* *}=\left(\int_{\mathrm{B}} \boldsymbol{x} \otimes(\boldsymbol{\omega} \times(\boldsymbol{\omega} \times \boldsymbol{x})) d \mu\right)_{h i}=\omega_{j} \omega_{i} I_{h j}-\omega_{j} \omega_{j} I_{h i} .
$$




\section{Introducing principal axes}

In order to display usable forms for expressions (31) and (32), let us specialize the orthonomal coordinates, with origin at the mass center of $\mathrm{B}$, in such a way that the coordinate axes are principal for the inertia tensor, i.e. the matrix $\left[I_{h k}\right]$ is diagonal and put $P_{1}=I_{11}, P_{2}=I_{22}, P_{3}=$ $I_{33}$. Such axes, assumed kinematically attached to B, are commonly used when writing code for the dynamics of $\mathrm{B}$ : as functions of time, the corresponding components of $\boldsymbol{\omega}$ may be taken as elements of the velocity function $t \mapsto u \in \mathbb{R}^{d}$ of the investigated mechanical system, with the considerable advantage that the corresponding elements of the $d \times d$ mass matrix make a $3 \times 3$ diagonal block, constant in time.

Let us first exhibit the components $T_{h i}^{*}$ from (31) (as usual, the first subscript refers to lines in the corresponding matrix).

For $h=i=1$, one restricts summations to $k=1$, since $I$ is diagonal; as $\varepsilon_{1 j 1}=0$, this yields $T_{11}^{*}=0$. Similarly $T_{22}^{*}=T_{33}^{*}=0$.

For $h=1$ and $i=2$, one restricts summations to $k=1$ and $j=3$ since otherwise $\varepsilon_{2 j 1}$ vanishes; as $\varepsilon_{231}=1$ this yields $T_{12}^{*}=\dot{\omega}_{3} P_{1}$. Similar calculations apply to all non diagonal terms, so

$$
\left[T_{h i}^{*}\right]=\left[\begin{array}{ccc}
0 & \dot{\omega}_{3} P_{1} & -\dot{\omega}_{2} P_{1} \\
-\dot{\omega}_{3} P_{2} & 0 & \dot{\omega}_{1} P_{2} \\
\dot{\omega}_{2} P_{3} & -\dot{\omega}_{1} P_{3} & 0
\end{array}\right]
$$

Let us now display the components $T_{h i}^{* *}$ from (32).

For $h=i=1$, one has $T_{11}^{* *}=\omega_{1}^{2} P_{1}-\left(\omega_{1}^{2}+\omega_{2}^{2}+\omega_{3}^{2}\right) P_{1}=-\left(\omega_{2}^{2}+\omega_{3}^{2}\right) P_{1}$ and similar expressions for $T_{22}^{* *}$ and $T_{33}^{* *}$.

If $h \neq i$, the term $I_{h i}$ in (32) vanishes so there remains $T_{12}^{* *}=\omega_{1} \omega_{2} P_{1}$, etc.. Summing up:

$$
\left[T_{h i}^{* *}\right]=\left[\begin{array}{ccc}
-\left(\omega_{2}^{2}+\omega_{3}^{2}\right) P_{1} & \omega_{1} \omega_{2} P_{1} & \omega_{1} \omega_{3} P_{1} \\
\omega_{2} \omega_{1} P_{2} & -\left(\omega_{3}^{2}+\omega_{1}^{2}\right) P_{2} & \omega_{2} \omega_{3} P_{2} \\
\omega_{3} \omega_{1} P_{3} & \omega_{3} \omega_{2} P_{3} & -\left(\omega_{1}^{2}+\omega_{2}^{2}\right) P_{3}
\end{array}\right]
$$

\section{Invoking rigid body dynamics}

In time-stepping numerics, some approximants of the derivatives $\dot{\omega}_{i}$ are commonly accessible through the increments of $\omega_{i}$, allowing one to exploit the expression of $\left[T_{h i}^{*}\right]$ above.

It should however be noticed that Euler's equations, which govern the dynamics of the rotation of the rigid body $\mathrm{B}$ about its center of mass $\boldsymbol{c}$, relate these derivatives to the vector moment $\boldsymbol{m}$ about $\boldsymbol{c}$ of the external efforts experienced by B. The moments of inertia of B about the three principal axes in use respectively equal $P_{2}+P_{3}, P_{3}+P_{1}, P_{1}+P_{2}$; assume them non zero, so that Euler's equations become

$$
\dot{\omega}_{1}=\frac{m_{1}}{P_{3}+P_{2}}+\frac{P_{3}-P_{2}}{P_{3}+P_{2}} \omega_{2} \omega_{3}
$$

and similarly for $\dot{\omega}_{2}$ and $\dot{\omega}_{3}$. One has to transport these expressions into $\left[T_{h i}^{*}\right]$; the resulting tensor may be decomposed into the sum of its symmetric part and its antisymmetric part. The latter is found to balance the antisymmetric part of -tens.mom.(ext.efforts) in (28). Anyway, both members of this equality are sure to be symmetric tensors, so that one may write equivalently

$$
M(\mathrm{~B})=- \text { sym.tens.mom.(ext.efforts) }+\operatorname{sym} \int_{\mathrm{B}} \boldsymbol{x} \otimes \boldsymbol{\gamma}(\boldsymbol{x}) d \mu(\boldsymbol{x}) .
$$

After reduction, the last term is found equal to the sum of two tensors with respective matrices: 


$$
\frac{1}{2}\left[\begin{array}{ccc}
0 & \frac{P_{1}-P_{2}}{P_{1}+P_{2}} m_{3} & \frac{P_{3}-P_{1}}{P_{3}+P_{1}} m_{2} \\
\frac{P_{1}-P_{2}}{P_{1}+P_{2}} m_{3} & 0 & \frac{P_{2}-P_{3}}{P_{2}+P_{3}} m_{1} \\
\frac{P_{3}-P_{1}}{P_{3}+P_{1}} m_{2} & \frac{P_{2}-P_{3}}{P_{2}+P_{3}} m_{1} & 0
\end{array}\right]
$$

and

$$
\left[\begin{array}{ccc}
-\left(\omega_{2}^{2}+\omega_{3}^{2}\right) P_{1} & 2 \frac{P_{1} P_{2}}{P_{1}+P_{2}} \omega_{1} \omega_{2} & 2 \frac{P_{1} P_{3}}{P_{1}+P_{3}} \omega_{1} \omega_{3} \\
2 \frac{P_{1} P_{2}}{P_{1}+P_{2}} \omega_{1} \omega_{2} & -\left(\omega_{3}^{2}+\omega_{1}^{2}\right) P_{2} & 2 \frac{P_{2} P_{3}}{P_{2}+P_{3}} \omega_{2} \omega_{3} \\
2 \frac{P_{1} P_{3}}{P_{1}+P_{3}} \omega_{1} \omega_{3} & 2 \frac{P_{2} P_{3}}{P_{2}+P_{3}} \omega_{2} \omega_{3} & -\left(\omega_{1}^{2}+\omega_{2}^{2}\right) P_{3}
\end{array}\right]
$$

\section{Spherical inertia}

The inertia tensor at the mass center $\boldsymbol{c}$ of B has the spherical symmetry if the mass distribution of the body possesses this symmetry, but also in less symmetrical situations such as the case of a homogeneous cube. Then, any coordinate frame with origin at $\boldsymbol{c}$ is principal and $P_{1}=P_{2}=P_{3}$, common value noted $P=I_{0} / 3$ where $I_{0}$ is the moment of inertia of $\mathrm{B}$ about the point $\boldsymbol{c}$. The matrix in (34) vanishes, while that in (35) becomes, after putting $\omega^{2}=\omega_{1}^{2}+\omega_{2}^{2}+\omega_{3}^{2}$,

$$
\left[\begin{array}{ccc}
P\left(\omega_{1}^{2}-\omega^{2}\right) & P \omega_{1} \omega_{2} & P \omega_{1} \omega_{3} \\
P \omega_{1} \omega_{2} & P\left(\omega_{2}^{2}-\omega^{2}\right) & P \omega_{2} \omega_{3} \\
P \omega_{1} \omega_{3} & P \omega_{2} \omega_{3} & P\left(\omega_{3}^{2}-\omega^{2}\right)
\end{array}\right]=P\left(\left[\omega_{i} \omega_{j}\right]-\omega^{2} \mathbf{1}\right) .
$$

Such becomes in this case the symmetric part of $\int_{\mathrm{B}} \boldsymbol{x} \otimes \boldsymbol{\gamma}(\boldsymbol{x}) d \mu(\boldsymbol{x})$.

\section{Two-dimensional models}

The rigid body $\mathrm{B}$ is then a plate, moving in a fixed plane to which the coordinate axis $\boldsymbol{c} x_{3}$ is normal. As before, the moving axes $\boldsymbol{c} x_{1}$ and $\boldsymbol{c} x_{2}$ are assumed principal for the inertia tensor of $\mathrm{B}$ at its mass center $\boldsymbol{c}$. If the plate is viewed a infinitely thin, the moment of inertia $P_{3}$ vanishes but what follows applies as well to a plate of nonzero thickness, B being then a solid of prismatic shape in the $x_{3}$ direction; in fact $P_{3}$ doesn't appear in the formulas to come.

Kinematically $\omega_{1}=\omega_{2}=0$ at every instant, while $\omega_{3}$ equals $\omega$, the angular velocity of the two-dimensional body. Then the above results simplify into

$$
\left[T_{h i}^{*}\right]=\left[\begin{array}{ccc}
0 & \dot{\omega} P_{1} & 0 \\
-\dot{\omega} P_{2} & 0 & 0 \\
0 & 0 & 0
\end{array}\right]
$$

and

$$
\left[T_{h i}^{* *}\right]=\left[\begin{array}{ccc}
-\omega^{2} P_{1} & 0 & 0 \\
0 & -\omega^{2} P_{2} & 0 \\
0 & 0 & 0
\end{array}\right]
$$


In expression (34), simplification arises from that two-dimensional dynamics involves $m_{1}=$ $m_{2}=0$, so it reduces to

$$
\left[T_{h i}^{*}\right]=\frac{1}{2} \frac{P_{1}-P_{2}}{P_{1}+P_{2}} m_{3}\left[\begin{array}{ccc}
0 & 1 & 0 \\
1 & 0 & 0 \\
0 & 0 & 0
\end{array}\right]
$$

As for expression (35), it becomes identical to (36).

Two-DIMENSIONAL ISOTROPY.- Isotropy here means that the inertia tensor of B at point $\boldsymbol{c}$ possesses the rotational symmetry about $\boldsymbol{c} x_{3}$ axis. This happens if the mass distribution of $\mathrm{B}$ possesses this symmetry but also if $\mathrm{B}$ is a homogeneous plate in the shape of a regular polygon. Then $P_{1}=P_{2}=I / 2$, with $I$ denoting the moment of inertia of B about $\boldsymbol{c} x_{3}$ and (33) reduces to

$$
M(\mathrm{~B})=- \text { sym.tens.mom. (ext.efforts })-\frac{\omega^{2} I}{2}\left[\begin{array}{lll}
1 & 0 & 0 \\
0 & 1 & 0 \\
0 & 0 & 0
\end{array}\right]
$$

\section{Percussions}

As recalled in the Introduction, the evolution of a granulate, hower slow it may seem at macroscopic observation level, usually involves microscopic crises in the course of which grains may collide. If these grains are modelled as perfectly rigid bodies, their contact interactions at collisional instants are not described as forces but as percussions. Even if only a single collision occurs in a cluster of contacting grains, percussions should be expected at every contact point. What precedes readily adapts to instants at which efforts are of percussional nature. Recall in this connection that CD computation consists in solving, on each time-step, the balance of momentum of the system, in which the forces manifest themselves through impulsions. Proper forces are retrieved after division by the step-length.

\section{What about stress transmission?}

*** à mettre peut-être à la suite du parag sur les point torques***

Let us consider a pack of spherical grains (or of circular ones in 2D models) contacting each other with dry friction. Assume that the peripheral grains are submitted to contact actions, arising from some confining boundaries or from an adjacent granular pack. Admit as usual that these actions consist of simple forces acting at isolated contact points. Since the point of application of each force is necessarily distinct from the center of the concerned spherical grain, one is naturally tempted to assert, as soon as the tangential component is nonzero, that the force "tends to make the grain rotate". The moment of the force about the grain center provides a natural measure of this tendency and it is sometimes declared that such a moment is "imparted" to the granular pack. This assertion may induce one to think that some moments (or couples) could be transmitted from peripheral grains into the rest of the pack, similarly to the transmission of forces. Actually, the transmission of forces is a radically different effect, governed by the fundamental principle that the two contact forces that a pair of grains exert upon each other are opposite. On the contrary, if the moments of these two forces about the centers of the respective spherical grains are calculated, they are found equal in case the grains have equal radii. If the grains are of different sizes, the ratio of the moments is anyway positive. This precludes the derivation, for a collection of grains, of balance equations which would involve the local moments in the same way as does the balance equations drawn for forces.

$* * * * * * * *$

Par contre, il semble raisonnable d'étudier la transmission de couples ponctuels si on se trouve en exercer à la frontière. Il faudrait adapter un programme Mac à la prise en compte des 
couples ponctuels et voir si on ne ferait pas apparaitre des chaines de moments semblables aux chaines de forces

$* * * * * * * * *$

voir [4] pour l'argument (contestable) que le tenseur de contrainte trouvé et son éventuelle dissymétrie dépend de la forme de la plage d'épreuve. La dissymétrie deviendrait ainsi importante dans les bandes de glissement.

\section{References}

[1] K. Bagi (1993) On the definition of stress and strain in granular assemblies through the relation between micro- and macro-level characteristics, in Powders and grains 93, C. Thornton (ed.), Balkema, Rotterdam, 117-121.

$[2] \_,(1996)$ Stress and strain in granular assemblies, Mechanics of materials 22, 165-177.

[3] _ , (1999) Microstructural stress tensor of granular assemblies with volume forces, J. Appl. Mech. 66, 934-936.

[4] J. P. Bardet \& I. Vardoulakis (2001) The asymmetry of stress in granular media, Int. J. Solids Struct. 38, 353-367.

[5] D. Caillerie (1995) Evolution quasistatique d'un milieu granulaire, loi incrémentale par homogénéisation, in Des géomatériaux aux ouvrages, Hermès, Paris, 53-80.

[6] B. Cambou \& D. Caillerie (2002) in (B. Cambou \& M. Jean, eds) Micromécanique des milieux granulaires, Hermes Sciences, Paris, Chap.2.

[7] C. Chree (1892) Changes in the dimension of elastic bodies due to given systems of forces, Cambridge Phil. Soc. Trans. 15, 313-337.

[8] J. Christoffersen, M. M. Mehrabadi \& S. Nemat-Nasser (1981) A micromechanical description of granular material behaviour, J. Appl. Mech. (Transactions of the ASME), 48, 339-344.

[9] D. Daudon, J. Lanier \& M. Jean (1997) A micromechanical comparison between experimental and numerical simulation of a bi-axial test on a 2D granular material, in (R. P. Behringer \& J. T. Jenkins, eds.) Powders and Grains '97, Balkema, Rotterdam, 219-222.

[10] G. de Saxcé, J. Fortin \& O. Millet (2004) About the numerical simulation of the dynamics of granular media and the definition of the mean stress tensor, to appear in Mechanics of Materials.

[11] A. Drescher \& G. de Josselin de Jong (1972) Photoelastic verification of a mechanical model for the flow of a granular material, J. Mech. Phys. Solids, 20, 337-351.

[12] S. F. Edwards (1998) The equations of stress in a granular material, Physica A, 249, 226231.

[13] J. Fortin, O. Millet \& G. de Saxcé (2002) Mean stress in a granular medium in dynamics, Mechanics Research Communications, 29, 235-240.

[14] J. Fortin, O. Millet \& G. de Saxcé (2003) Construction of an average stress tensor for a granular medium, European J. Mech. A/Solids, 22, 567-582.

[15] M. Frémond (2002) Non-smooth thermomechanics, Springer. 
[16] G. Friedlander \& M. Joshi (1998) Introduction to the theory of distributions, Cambridge University Press.

[17] P. Germain (1973) The method of virtual power in continuum mechanics. Part 2: microstructures, SIAM J. Appl. Math. (1973), 556-575.

[18] J. D. Goddard (1986) Microstructural origins of continuous stress fields - A brief history and some unresolved issues, in: (D. De Kee, ed.) Recent developments in structured continua, Longmans, 179-208.

[19] _ , (1998) Continuum modelling of granular assemblies, in H. J. Herrmann et al. (eds.), Physics of Dry Granular Materials, Kluwer, Dordrecht/Boston/London, 1-24.

[20] A. E. Green \& R. S. Rivlin (1964) Simple force and stress multipoles, Arch. Rational Mech. Anal., 16, 325-353.

[21] Gurtin, M. E.(1972) The linear theory of elasticity, in: (Flügge, ed.) Encyclopedia of Physics, vol. VIa/2, Springer-Verlag, Berlin.

[22] N. P. Kruyt \& L. Rothenburg (1996) Micromechanical definition of the strain tensor for granular materials, J. Appl. Mech., 118, 706-711.

[23] J. Lanier \& M. Jean (2000) Experiments and numerical simulations with 2D disks assembly, Powder Technology, 109, 206-207.

[24] E. Leflaive, M. Khay, \& J.-C. Blivet (1983) Un nouveau matériau: le Texsol, Bulletin de Liaison des Laboratoires des Ponts et Chaussées, 125, 105-114.

[25] R. I. Leine \& Ch. Glocker (2003) A set-valued force law for spatial Coulomb-Contensou friction, European Journal of Mechanics A/Solids 22, 193-216.

[26] Ph. Liausu \& I. Juran (1995) Texsol: material properties and engineering performance, Transportation Research Record, n 1474, 3-12.

[27] S. Luding (2002) From microscopic simulations to macroscopic material behavior, Computer Physics Communications, 147, 134-140.

[28] M. Louge (2003) Model for dense granular flows down bumpy inclines, Phys. Rev. E, 67, 061303, 1-10.

[29] A. E. H. Love (1944) A treatise on the mathematical theory of elasticity (reprint of the 4th. edition, 1927), Dover Publications, New York.

[30] W. Meftah, P. Evesque, J. Biarez, D. Sornette \& N. E. Abriak (1993) Evidence of local "seisms" of microscopic and macroscopic stress fluctuations during the deformation of a packing of grains, in Powders and grains 93, C. Thornton (ed.), Balkema, Rotterdam, 173-178.

[31] B. Miller, C. O'Hern \& R. P. Behringer (1996) Stress fluctuations for continuously sheared granular materials, Phys. Rev. Letters, 77(15), 3110-3113.

[32] J. J. Moreau (1988) Unilateral contact and dry friction in finite freedom dynamics, in: (J. J. Moreau \& P. D. Panagiotopoulos, eds.) Nonsmooth Mechanics and Applications, CISM Courses and Lectures, Vol. 302, Springer-Verlag, Wien New York, 1-82.

[33] — (1988) Bounded variation in time, in: (J. J. Moreau, P. D. Panagiotopoulos \& G. Strang, eds.) Topics in Nonsmooth Mechanics, Birkhäuser, Basel, Boston, Berlin, 1-74. 
[34] _ (1988) Free boundaries and non-smooth solutions to some field equations: variational characterization through the transport method, in: (J. P. Zolésio, ed.) Boundary Control and Boundary Variations, Lecture Notes in Comput. Sci., 100, Springer-Verlag, New York, Berlin, Heidelberg, pp. 235-264.

[35] — - (1989) An expression of classical dynamics, Ann. Inst. H. Poincaré Anal. Non Linéaire, 6 (suppl.), 1989, pp. 1-48. Volume also available as: (H. Attouch, J.-P. Aubin, F. Clarke \& I. Ekeland, eds.) Analyse Non Linéaire, Gauthier-Villars, Paris.

[36] _ (1997) Numerical investigation of shear zones in granular materials, in P. Grassberger and D. E. Wolf (eds.), Friction, Arching, Contact Dynamics, World Scientific, Singapore, 233247.

[37] — An introduction to unilateral dynamics, in: (M. Frémond \& F. Maceri, eds.) Novel approaches in Civil Engineering, Lecture Notes in Applied and Computational Mechanics, vol. 14, Springer-Verlag, 2004, pp. 1-46.

[38] G. Morel \& M. Khay (1990) Propriétés mécaniques du matériau sol-fibres Texsol, Bulletin de Liaison des Laboratoires des Ponts et Chaussées, 83-93.

[39] A. Ngadi, J. Rajchenbach, E. Clément \& J. Duran (1997) Intermittences in the compression process of a model granular medium, in Powders and grains 97, R. P. Behringer \& J. T. Jenkins (eds.), Balkema, Rotterdam, 321-324.

[40] C. Nouguier, C. Bohatier, J. J. Moreau, F. Radjai (2000) Force fluctuations in a pushed granular material, Granular matter, 2 (4), 171-178.

[41] C. A. Truesdell \& R. A. Toupin (1960) The Classical Field Theories, Handbuch der Physik, Springer, Berlin-Göttingen-Heidelberg.

[42] F. Radjai \& S. Roux (2002) Turbulentlike fluctuations in quasistatic flow of granular media, Phys. Rev. Letters, 89, Nr 6.

[43] L. Rothenburg and A. P. S. Selvadurai (1981) A micromechanical definition of the Cauchy stress tensor for particulate media, in A. P. S. Selvadurai (ed.) Proc. Int. Symp. on the Mechanical Behavior of Structured Media, Ottawa, Canada.

[44] L. Staron, J. P. Vilotte \& F. Radjai (2004) Multiscale analysis of the stress state in a granular slope in transition to failure, to appear.

[45] J. Weber (1966) Recherches concernant les contraintes intergranulaires dans les milieux pulvérulents, Cahiers du Groupe Français de Rhéologie, 2, 161-170 (a more detailed account may be found under the same title in Bulletin de Liaison des Ponts et Chaussées, 20, juil.-août 1966, pp. 3-1 to $3-20)$

[46] H. P. Zhu \& A. B. Yu (2002) Averaging method of granular material, Phys. Rev. E, 66, 021302 (10 pages). 